\title{
An Experimental Test Procedure for Validation of Stiffness Model: A Case Study for R-CUBE Parallel Mechanism
}

\author{
İbrahimcan Görgülü ${ }^{1}$, M. İ. Can Dede ${ }^{1}$, and Giuseppe Carbone ${ }^{2}$ \\ 1 Izmir Institute of Technology, Izmir, Turkey, \\ ibrahimcangorgulu, candede@iyte.edu.tr, \\ 2 DIMEG, University of Calabria, Rende, Italy \& Technical University of \\ Cluj-Napoca, Cluj-Napoca, Romania, \\ giuseppe.carbone@unical.it
}

\begin{abstract}
Haptic device manipulators are used for generating haptic feedback. This feedback is composed of force which is regulated with respect to motion information. Accurate generation of the feedback requires exact position acquisition of the end-effector. Due to the compliant bodies of a manipulator, a stiffness model is needed to predict this position. Previously, Virtual Joint Method was adopted to obtain the stiffness model of an R-CUBE parallel haptic mechanism. In this paper, experimental test setup and experimental procedure are described for validating this stiffness model, its engineering feasibility and soundness of the proposed model.
\end{abstract}

Keywords: Virtual Joint Method, Parallel Mechanism, Experimental Stiffness Validation

\section{Introduction}

A haptic device reflects force/motion information to a user to generate haptic stimuli [1]. This information is generated by making use of the end-effector position. It is common to use forward kinematics and joint sensor data (e.g. encoders) to compute this position. However, joint sensors cannot detect the compliant displacements of compliant manipulators. Hence, the kinematic model is not sufficient to compute the actual position. Correspondingly, force/torque information to be displayed to the user is generated inaccurately. Since the accuracy of haptic stimulus is related to the accurate acquisition of position information, a stiffness model is required to estimate the actual end-effector position.

A stiffness model is an ideal mathematical model. However, the manufactured manipulators may have imperfectness in their geometry, sub-component, and material. These imperfectnesses causes divergence in compliant deflections between the model and the manipulator. In order to reduce this divergence, the stiffness model has to be verified and modified via experiments. A general procedure is described in [2] to obtain an exact stiffness model. Another procedure is proposed in [3] to verify and modify the stiffness model of robotic systems 
experimentally. In [4], stiffness model of a parallel manipulator that has sagging cables was experimentally verified.

In an experiment of stiffness mapping, the manipulator is positioned at several poses or followed a pre-defined trajectory with a relatively low speed. Simultaneously, an external wrench is applied to the end-effector. Meanwhile, compliant displacements are measured. The measurement instrument type defines the type of experiment method. Some merits and standards on the measurement of geometric errors and instrument types are given in [5]. Comparison of position measurement sensors are given in [6]. These position sensors may be used to obtain compliant displacements for stiffness experiments. Also, they may or may not require contact with the test subject. In addition, some of these sensors directly measure the end-effector position while other ones measure indirectly. This indirect measurement requires a model that relates the measured data in sensor-space to the compliant displacement in task space.

In [7], and [8], a camera is used for contactless and direct measurement approach. In this method, a reference picture is attached to the end-effector. Then, the pose information of this picture is computed via image processing. Depending on the change in the pose of the reference image, the compliant displacement is computed. The accuracy of the results in this technique is dependent on the lighting conditions, and the resolution of the camera. However, the computation cost is relatively higher compared to the other methods.

In [9], coordinate measuring machines (CMM) are used to measure the position via its probe directly. Hence, CMM needs physical contact. This may expose additional external wrench on the end-effector. Nevertheless, if this contact force is relatively smaller than the designated minimum force output, then the wrench of the probe cannot neglected.

An indirect measurement approach is proposed in [10]. In this study, a cablebased parallel manipulator called Milli-CaTraSys is coupled with the mobile platform of CaPaMan parallel manipulator. Then, the change in mobile platform position/orientation is computed by measuring the displacements of cables. Although this system is highly accurate, it requires precise calibration to begin the tests.

In [11], and [12] laser distance sensors are used to measure the end-effector position. This laser sensor demands a reference surface to observe the displacement. One drawback of the laser sensor is that it can only measure the translational displacements along the axis of the laser beam. Therefore, several of them must be used to get the translational and rotational deflections. Despite this drawback, a laser sensor is easy to use, has high resolution, and gives accurate and precise data.

Virtual Joint Method (VJM) is one of the methods to obtain an analytical stiffness model. It has a relatively shorter computation time. Therefore, it can run in a real-time control loop [13]. In this regard, this study is devoted to the construction of a test setup for the experimental validation of the VJM model of an R-CUBE mechanism [14]. A laser sensor is used to measure the compliant displacements directly from the mobile platform. First, a short description of 
the R-CUBE mechanism is given in Section 2. In Section 3, VJM model of the manipulator is briefly explained. In Section 4 and 5, experimental setup and procedure are described. In Section 6 results of experiments are illustrated and in Section 7 conclusion is addressed.

\section{R-CUBE Mechanism}

The R-CUBE manipulator is introduced by [14]. This manipulator comprises only revolute joints. It has 3 serial chains. Each chain actuates one of the translational degree of freedom (DOF) of the mobile platform. In Fig. 1 kinematic model of the manipulator is illustrated.

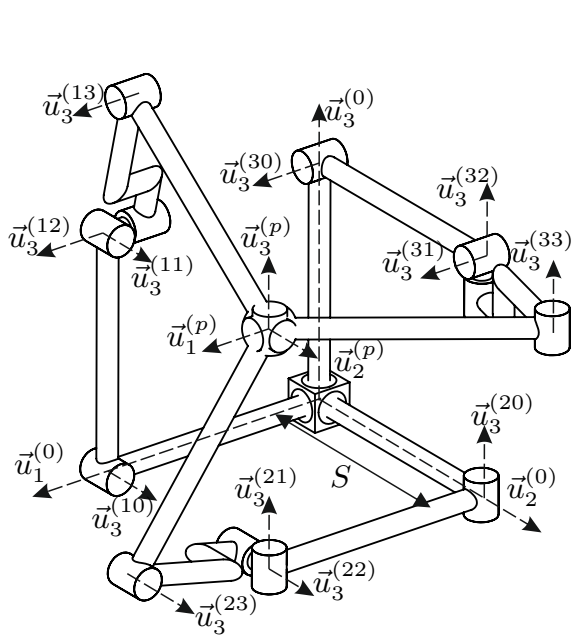

a)

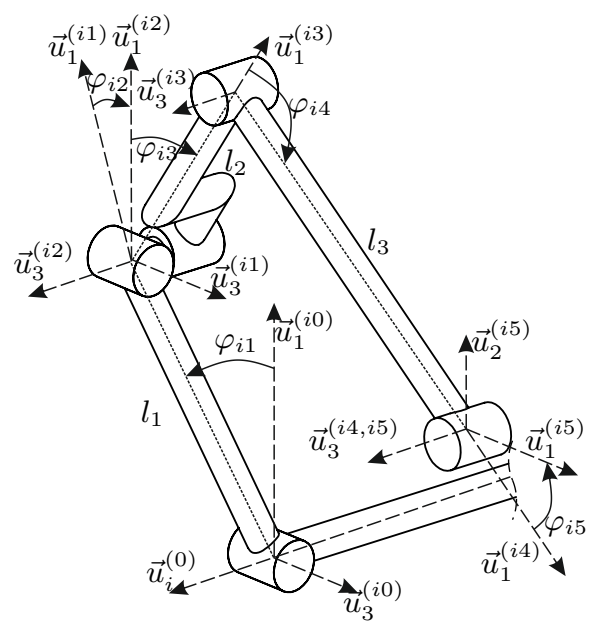

b)

Fig. 1. Kinematic sketches and frames of the R-CUBE mechanism: a) the manipulator, b) variables of $i^{\text {th }}$ serial chain where $i$ is one of the serial chains.

In Fig. 1 a), initial frames of the serial chains are located on $\vec{u}_{k}^{(0)}$ orthogonal unit vectors along $k^{\text {th }}$ axis for $k=1,2,3 . \vec{u}_{k}^{(i j)}$ denotes the unit vector belonging $i^{\text {th }}$ serial chain and $j^{\text {th }}$ frame as shown in Fig. 1 b). Due the kinematic constraints, $\vec{u}_{k}^{(0)} \| \vec{u}_{k}^{(p)}$ where $p$ is mobile platform frame. Also, $\vec{u}_{3}^{(15)}, \vec{u}_{3}^{(25)}, \vec{u}_{3}^{(35)}$ are always aligned with $\vec{u}_{1}^{(35)}, \vec{u}_{1}^{(15)}, \vec{u}_{1}^{(25)}$ vectors, respectively. The forward kinematics of the mechanism is simply given as:

$$
r_{i}=S+l_{1} \sin \varphi_{i 1} \text { for } i=1,2,3 \text { and } \bar{r}=\left[\begin{array}{ll}
r_{1} & r_{2} r_{3}
\end{array}\right]^{T}
$$

where $S$ is a constant length between the $0^{\text {th }}$ frame and $\vec{u}_{3}^{(i 0)}$ axis. $\bar{r}$ denotes the column matrix form of position vector with respect to the origin.

\section{Stiffness Model}

VJM is a lumped model approach based on the Jacobian matrix mapping and the virtual work principle. The stiffness information of a link is lumped on virtual 
springs defined on the virtual joints at the tip of this link. A lumped model approach for parallel manipulators is described for the first time in [15]. In [16], VJM approach is systematized for parallel manipulators, and the effects of passive joints on stiffness are considered.

In this regard, the compliant kinematic model of R-CUBE mechanism is obtained through virtual joints. Given in Fig. 2, compliance model of a single serial kinematic chain of R-CUBE is presented. Each virtual joint has 6 DOF that are 3 translations and 3 rotations. Kinematics of a virtual joint is defined as:

$$
\boldsymbol{H}_{v}\left(\bar{\theta}_{i j}\right)=\boldsymbol{T}_{1}\left(\theta_{i j}^{1}\right) \boldsymbol{T}_{2}\left(\theta_{i j}^{2}\right) \boldsymbol{T}_{3}\left(\theta_{i j}^{3}\right) \boldsymbol{R}_{1}\left(\theta_{i j}^{4}\right) \boldsymbol{R}_{2}\left(\theta_{i j}^{5}\right) \boldsymbol{R}_{3}\left(\theta_{i j}^{6}\right)
$$

where $\boldsymbol{H}_{v}$ denotes the homogeneous transformation matrix, $\boldsymbol{T}_{k}$ and $\boldsymbol{R}_{k}$ denote homogeneous transformation matrices for pure translation along and pure rotation about $\vec{u}_{k}^{\text {th }}$ axis for $k=1,2,3 . \bar{\theta}_{i j}$ is virtual joint variables that includes the translational and rotational DOF. Superscripts of $\theta_{i j}$ denote element number.

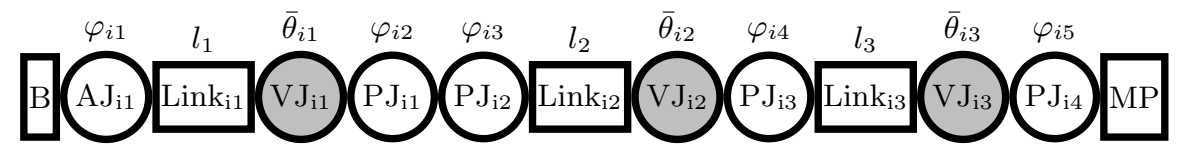

Fig. 2. Compliant kinematics of the manipulator where AJ is active joint, $\mathrm{PJ}$ is passive joint, VJ is virtual joint, MP is mobile Platform, and B is base.

Stiffness matrix of $i^{\text {th }}$ serial chain, $\boldsymbol{K}_{C i}$, is obtained via the compliant kinematics in Fig. 2. This matrix is given in Cartesian space coordinates as derived in [13]. Then, Cartesian stiffness matrix of the manipulator, $\boldsymbol{K}_{C}$, is computed as $\boldsymbol{K}_{C}=\sum_{i=1}^{3} \boldsymbol{K}_{C i}$. Components of $\boldsymbol{K}_{C}$ is given in Eq. 3 for small amount of deflections. This matrix must be re-computed if external load $\left|\bar{F}_{\text {ext }}\right|>>0$.

$$
\boldsymbol{K}_{C}=\left[\begin{array}{cccccc}
K_{C_{1}}^{(11)} & 0 & 0 & 0 & K_{C_{1}}^{(15)} & K_{C_{1}}^{(16)} \\
0 & K_{C_{2}}^{(22)} & 0 & K_{C_{2}}^{(24)} & 0 & K_{C_{2}}^{(26)} \\
0 & 0 & K_{C_{3}}^{(33)} & K_{C_{3}}^{(34)} & K_{C_{3}}^{(35)} & 0 \\
0 & K_{C_{2}}^{(24)} & K_{C_{3}}^{(34)} & K_{C_{2}}^{(44)}+K_{C_{3}}^{(44)} & K_{C_{3}}^{(45)} & K_{C_{2}}^{(46)} \\
K_{C_{1}}^{(15)} & 0 & K_{C_{3}}^{(35)} & K_{C_{3}}^{(45)} & K_{C_{1}}^{(55)}+K_{C_{3}}^{(55)} & K_{C_{1}}^{(56)} \\
K_{C_{1}}^{(16)} & K_{C_{2}}^{(26)} & 0 & K_{C_{2}}^{(46)} & K_{C_{1}}^{(56)} & K_{C_{1}}^{(66)}+K_{C_{2}}^{(66)}
\end{array}\right]
$$

\section{Experimental Setup}

The test setup is composed of a 3D printed R-CUBE manipulator, a laser range sensor, a reference plane to reflect the laser light, and a pulley-guide system to apply an external wrench to the manipulator. In Fig. 3 a), the constructed test setup is shown. In Fig. 3 b), a sketch of the CAD model of the test setup is given denoting some components of the setup.

The prototype is manufactured with Polylactic acid (PLA) material via a 3D printer. Each 3D printed part has 15\% infill. PLA material results in relatively larger compliant displacements compared to composite and metals. However, this behavior of PLA makes it more convenient to be used in stiffness evaluation tests 


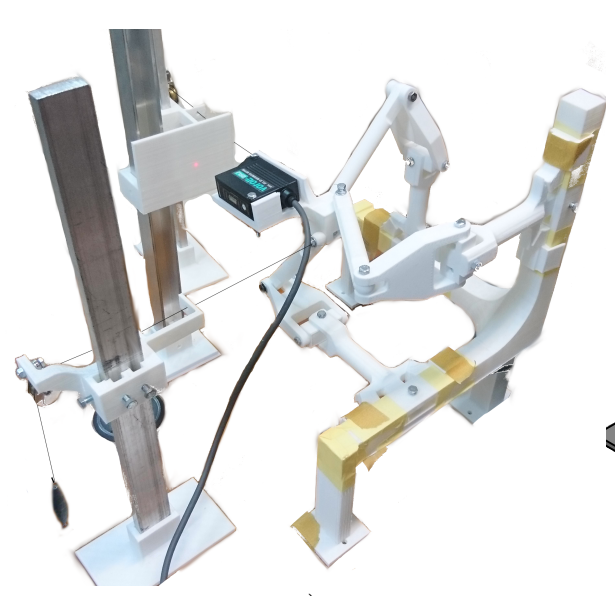

a)

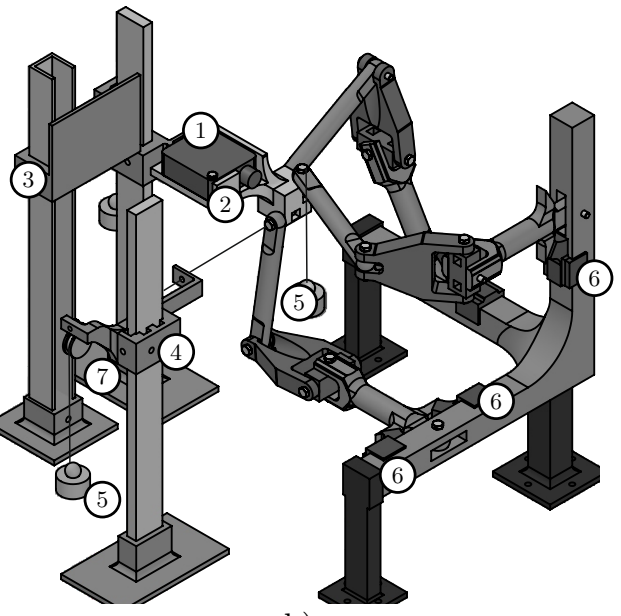

b)

Fig. 3. Test setup in different. a) Built setup, b) CAD model where 1: Laser sensor, 2: Mobile platform, 3: Reference plane, 4: Guide, 5: Weights, 6: Stoppers, 7: Pulley.

because it is easier to measure larger compliant displacements (from 0.1 to $1 \mathrm{~mm}$ ) with higher accuracy. In this range of displacements, the sensor noise during the measurements becomes insignificant and thus, it can be neglected. PLA differs from metals by being thermoplastic material that has visco-elastic properties. This behavior requires the consideration of the rate of applying the external wrenches. If there is a quick loading-unloading scenario such as operation in high frequencies, viscous forces become more dominant than the elastic forces. Hence, a damping model is also required in addition to stiffness model. However, the study focuses on structural stiffness. Hence, the measurements are obtained when the manipulator is in its final position and stabilized, and thus, the viscous forces are abolished.

M5 screws are used at the joint structures to connect the links. Screw connection increases the static friction in the joints. In the stiffness model, it was assumed that there was no friction in the passive joints. Accordingly, this friction induces errors in the stiffness model. Nonetheless, these joints have less joint clearance compared to a joint structure with bearings. This property minimizes the level of uncertainty in the manipulator's pose. The friction force, on the other hand, is smaller compared to applied external wrenches. Hence, neglecting the friction forces do not generate a significant error.

A Nippon brand LAS-5010V model laser range sensor is used for measuring the compliant displacements. Laser range sensor signals are acquired via a National Instruments data acquisition card. These distances are measured from the reference plane. This reference plane is located depending on the test poses of the manipulator. 
The intention is to measure the translational compliant displacements along $\vec{u}_{k}^{(p)}$ for $k=1,2,3$. For this purpose, the mobile platform of R-CUBE mechanism is designed to align the laser sensor with motion axes. The orientation of the mobile platform is changed to measure compliant displacements along the respective motion axis. That way, compliant translational displacement parallel to $\vec{u}_{k}^{(p)}$ for $k=1,2,3$ axes can be determined, separately. The orientation of the mobile platform changes due to the weight of the laser range sensor and external wrench. However, these orientation changes are assumed to be close to zero. Hence, orientation effects on the measurements are neglected since a relative measurement is done for compliant displacements.

External force vectors are aligned with $\vec{u}_{1}^{(p)}, \vec{u}_{2}^{(p)}$ and $\vec{u}_{3}^{(p)}$ axes as shown in Fig. 4. This alignment ensures that there is no external torque input to the manipulator. These external forces are generated by calibrated weights hung by a cable-pulley system. One end of the cable is connected to a mass, and the other end is connected to last frames of $1^{\text {st }}$ and $2^{\text {nd }}$ serial chains. This cable passes through a pulley and the 2 holes of the guide system. Location and the height of the guide system are adjusted so that the cable passes through the centers of these holes, so it is aligned with the respective motion axis. Guides allow us to adjust the height so that this test system can be used for any test

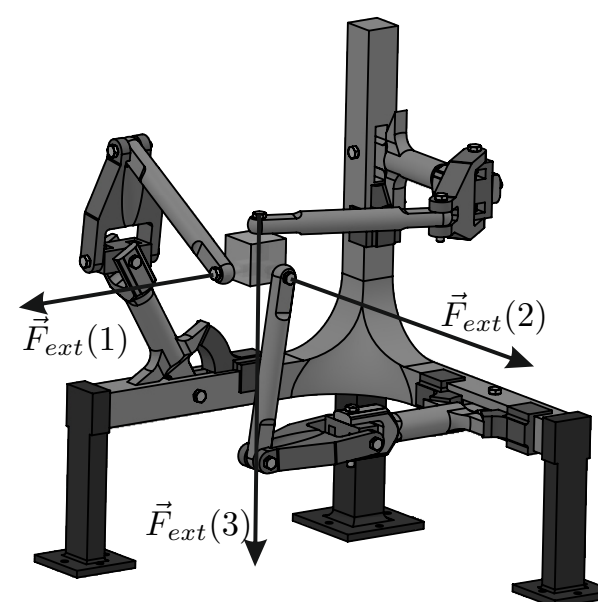

Fig. 4. External wrench components and their application points. pose.

Since hung masses generate the external forces, force vectors along $\vec{u}_{1}^{(p)}$ and $\vec{u}_{2}^{(p)}$ are always applied in a positive direction. The external force vector along $\vec{u}_{3}^{(p)}$ is always in the negative direction and aligned with the gravity vector. These directions of external force vectors do not change during the test procedure. Hence, the validation of stiffness model is conducted only for this force combination.

The manipulator should be tested throughout its workspace to capture its stiffness characteristics. These tests are often conducted by following a test trajectory in the workspace with low speeds. However, measurements in such a scenario include the stiffness property of the actuator. Since the objective of this study is to obtain structural stiffness, the mobile platform should be positioned and locked at several discrete poses in the workspace. Unfortunately, there are infinitely many discrete poses in the workspace. However, some of these poses have unique stiffness characteristics. By testing the manipulator at these poses, desired stiffness properties may be obtained. Therefore, some critical poses are 
identified to run the experiment. 27 poses are determined in total depending on the fully folded, fully expanded, and nominal poses of active links. 10 critical test poses (TPs) are selected for experiments. These TPs are given in Table 1. Later, the results at these TPs may be used to generate full stiffness model of the manipulator via interpolation. The manipulator is positioned to these poses manually by the help of mechanical limits on the first links and stopper parts. Stoppers are designed to lock the manipulator when the active joint is located at $0^{\circ}$ or $\pm 30^{\circ}$.

Table 1. Active joint values (AJV) of selected TPs, $\mathbf{T P}=\mathbf{T P}\left(\boldsymbol{\varphi}_{\mathbf{1 1}}, \boldsymbol{\varphi}_{\mathbf{2 1}}, \boldsymbol{\varphi}_{\mathbf{3 1}}\right),-30 \Rightarrow$ $-30^{\circ},+30 \Rightarrow+30^{\circ}, 0 \Rightarrow 0^{\circ}$

\begin{tabular}{c|cccccccccc}
\hline AJV & TP1 & TP2 & TP3 & TP4 & TP5 & TP6 & TP7 & TP8 & TP9 & TP10 \\
\hline $\boldsymbol{\varphi}_{\mathbf{1 1}}$ & -30 & 0 & +30 & 0 & 0 & 0 & 0 & +30 & +30 & 0 \\
$\boldsymbol{\varphi}_{\mathbf{2 1}}$ & -30 & 0 & +30 & 0 & 0 & +30 & -30 & -30 & -30 & -30 \\
$\boldsymbol{\varphi}_{\mathbf{3 1}}$ & -30 & 0 & +30 & +30 & -30 & +30 & -30 & -30 & +30 & +30 \\
\hline
\end{tabular}

\section{Experimental Procedure}

In this section, the experimental procedure is described. First, the experiment factors are determined to conduct a comprehensive experiment. These factors are laser range sensor orientation $(a)$, test pose $(b)$, attached mass $(c)$, and recorded sample $(d)$, where $a, b, c, d$ are their labels. The laser sensor orientation is aligned with motion axes to measure compliant deflections in these directions. A test pose is selected from Table 1. In each test pose, the manipulator is locked via stoppers. Then, the masses are attached to end-effector by using the pulley-guide system for each motion axis. Different masses enable to observe the effect of different wrenches on compliant deflections. In each set value of $a, b, c$, a measurement is recorded for 3 times and each recording is labeled with $d$ parameter. This repetitive recording is done to increase confidence on the measured data. Table 2 shows the possible values of these factors and their ranges. These values are presented with respect to their selection order.

Table 2. Experiment factors.

\begin{tabular}{r|c|l}
\hline Factors & Range of Labels & Values of Factors in Order \\
\hline Laser orientation, $\mathbf{a}$ & $1-3$ & $\vec{u}_{1}^{(0)}, \vec{u}_{2}^{(0)}, \vec{u}_{3}^{(0)}$ \\
Test pose, $\mathbf{b}$ & $1-10$ & $\mathrm{TP} 1-\mathrm{TP} 10$ \\
Attached mass, c & $1-4$ & $285 \mathrm{~g}, 185 \mathrm{~g}, 85 \mathrm{~g}, 0 \mathrm{~g}$ \\
Sample number, d & $1-3$ & $1,2,3$ \\
\hline
\end{tabular}

The experimental test procedure starts by setting all factor labels to 1 ( $a=$ $1, b=1, c=1, d=1)$. Hence, the initial test configuration measures the deflections along $\vec{u}_{1}^{(p)}$ axis at TP1 with $285 \mathrm{~g}$ forces in all directions. After the manipulator is stabilized (when the visco-elastic behavior diminishes), the distance between the laser sensor and reference plane is recorded with label $d=1$. Next, masses are removed, and reattached 2 more times to record the data for $d=2$, and $d=3$. This attach-and-remove procedure prevents incorrect data 
measurement that might be caused by nonlinear effects at joints such as friction and joint clearance. Then, $185 \mathrm{~g}, 85 \mathrm{~g}$, and $0 \mathrm{~g}$ masses are attached for $c=2,3,4$ and 3 , and measurements are recorded for $d=1,2,3$. This higher to lower loading sequence for $c$ ensures that the mechanism is in its final compliant pose, and there is no displacement due to joint clearance. Notice that, when $c=4$, there is no mass attached. This measurement determines the distance to the reference plane for the unloaded case. Hence, this value is used to subtract the offset value of the sensor data to compute compliant displacement. Then, same procedure is conducted for TP2 to TP10 for $b=2,3, \ldots, 10$ and in different motion axes for $a=2,3$ for measurement at different poses in different directions. A flow chart of the procedure is described in Fig. 5.

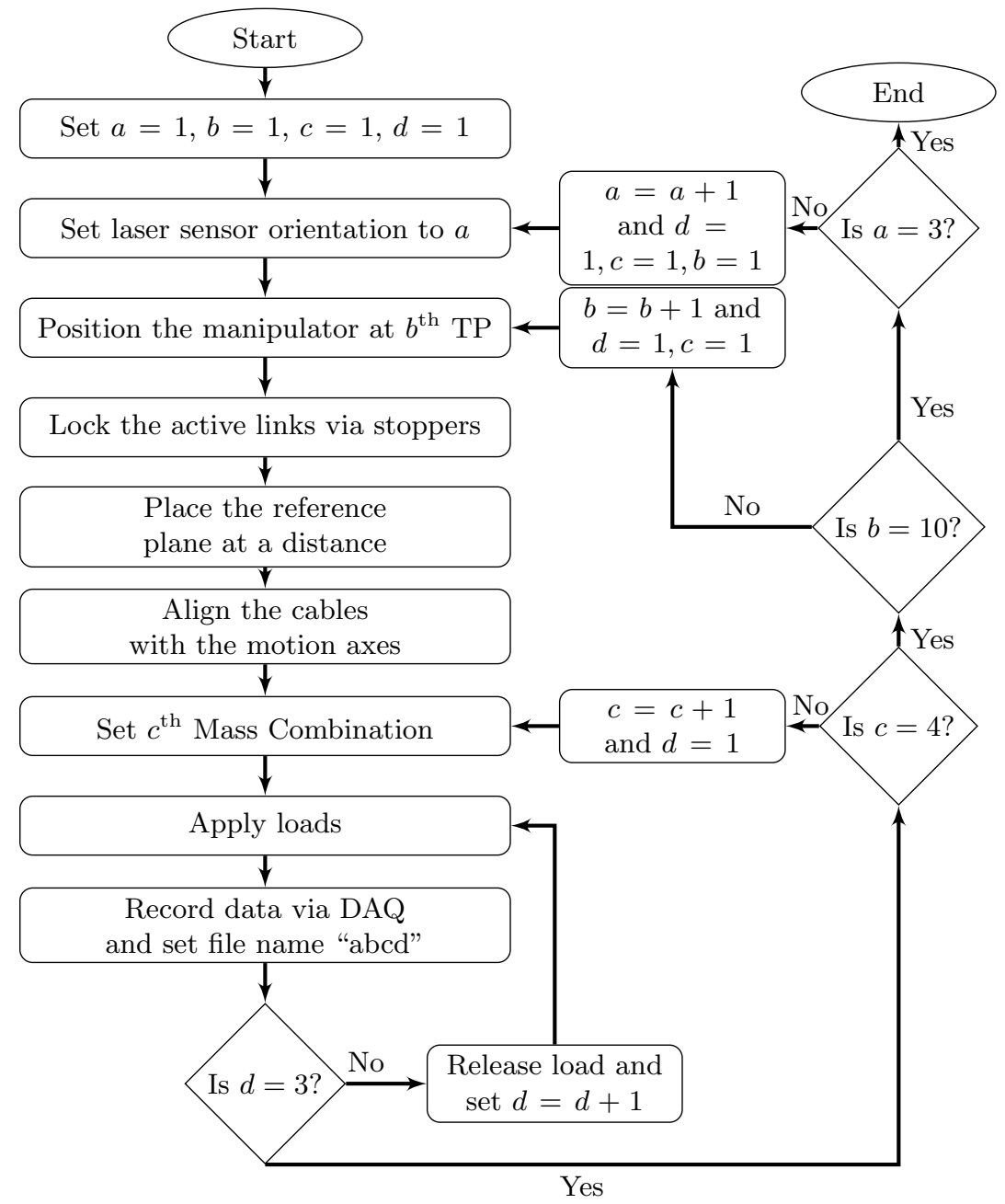

Fig. 5. Flow chart of experimental procedure. 


\section{Test Results and Comparison with the VJM model}

Obtained raw data (voltage readings) from laser range sensor are recorded in LabVIEW. Then, they are post-processed in MATLAB for conversion of the raw data into distance information. The compliant displacements are computed by subtracting the unloaded distance from loaded distances. The numerical results are given in Table 3 for deflections along $\vec{u}_{1}, \vec{u}_{2}$, and $\vec{u}_{3}$ motion axes, respectively.

Table 3. Absolute values of experimental deflection measurements along $\vec{u}_{1}, \vec{u}_{2}$, and $\vec{u}_{3}$ in $\mathrm{mm}$.

\begin{tabular}{|c|c|c|c|c|c|c|c|c|c|c|}
\hline & TP1 & TP2 & TP3 & TP4 & TP5 & TP6 & TP7 & TP8 & TP9 & TP10 \\
\hline$\vec{u}_{1} 85 \mathrm{~g}$ & 0,23 & $\overline{0,34}$ & 0,40 & 0,51 & 0,19 & 0,72 & 0,18 & $\overline{0,26}$ & 0,54 & 0,92 \\
\hline$\vec{u}_{1} 185 \mathrm{~g}$ & 0,34 & 0,76 & 0,94 & 1,02 & 0,41 & 1,44 & 0,46 & 0,47 & 1,05 & 1,70 \\
\hline$\vec{u}_{1} 285 \mathrm{~g}$ & 0,51 & 1,22 & 1,39 & 1,64 & 0,85 & 2,23 & 0,87 & 0,71 & 1,47 & 2,46 \\
\hline$\vec{u}_{2} 85 \mathrm{~g}$ & 0,37 & 0,34 & 0,46 & 0,47 & 0,46 & 0,56 & 0,13 & 0,40 & 0,92 & 0,77 \\
\hline$\vec{u}_{2} \quad 185 \mathrm{~g}$ & 0,45 & 0,58 & 0,90 & 0,93 & 0,83 & 0,92 & 0,60 & 0,82 & 1,70 & 1,48 \\
\hline$\vec{u}_{2} 285 \mathrm{~g}$ & 0,66 & 0,85 & 1,14 & 1,25 & 1,23 & 1,35 & 0,89 & 1,30 & 2,50 & 2,01 \\
\hline$\vec{u}_{3} 85 \mathrm{~g}$ & 0,02 & 0,63 & 0,68 & 0,88 & 0,38 & 0,87 & 0,41 & 0,17 & 0,80 & 0,56 \\
\hline$\vec{u}_{3} 185 \mathrm{~g}$ & 0,23 & 1,66 & 1,62 & 1,70 & 0,81 & 1,74 & 0,63 & 0,51 & 1,63 & 1,29 \\
\hline$\vec{u}_{3} 285 \mathrm{~g}$ & 0,39 & 2,34 & 2,30 & 2,44 & 1,33 & 2,16 & 0,81 & 0,77 & 2,56 & 2,15 \\
\hline
\end{tabular}

In stiffness modeling, it is assumed that the compliant deflections are linearly proportional to applied force/torque. Hence, force to deflection ratio (the stiffness) is constant for small deflections. If the measured deflections are normalized with respect to their corresponding wrenches, the resultant ratio of different deflections and forces should be equal in the same test pose. When the compliant deflection results with $85 \mathrm{~g}, 185 \mathrm{~g}$, and $285 \mathrm{~g}$ of loads are plotted with respect to test poses, they should overlap on each other in normalized space. In an ideal case, the VJM model and the experiment measurements should also overlap in normalized space. However, a perfect overlap should not be expected due to manufacturing errors, joint clearances, etc. Yet, a correlation is still expected with minor errors. The normalization of VJM deflections and experimental ones are achieved by dividing each deflection set to their maximum value. Also, overall deflections are computed and normalized for comparison. Fig. 6 shows the VJM and experimental results in normalized space. It can be observed that measurements are a close correlation. However, VJM deflections are less correlated with the experiment results as expected.

The best fit between VJM and experiments are observed for $\vec{u}_{1}$ deflections, as shown in Fig. 6 a). Except for TP4 and TP10, VJM exhibits identical deflection behavior with the experiments. The maximum error between VJM and experiment is observed at TP3 and TP10. However, TP1, TP2, TP4, TP5, TP6, and TP9 results are close to each other.

$\vec{u}_{2}$ deflections are shown in Fig. 6 b). It has been observed that the experimental and VJM deflection data have a similar trend. In both data set, deflections increase or decrease about in same TPs. TP1 and TP2 results of VJM and experiment have the minimum relative error compared to the other TPs. VJM-experiment deflections in other TPs highly differ from each other. 


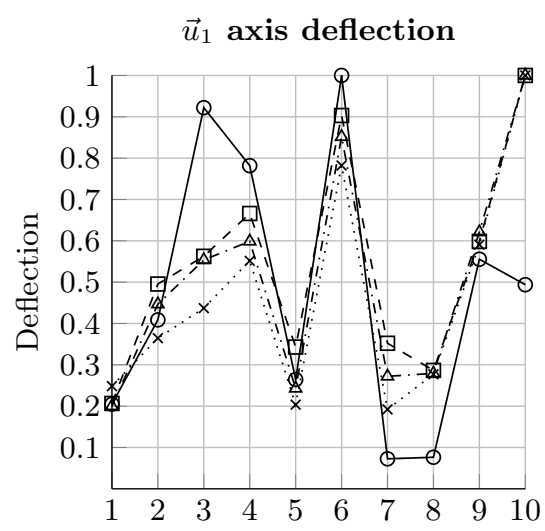

(a) Test Pose

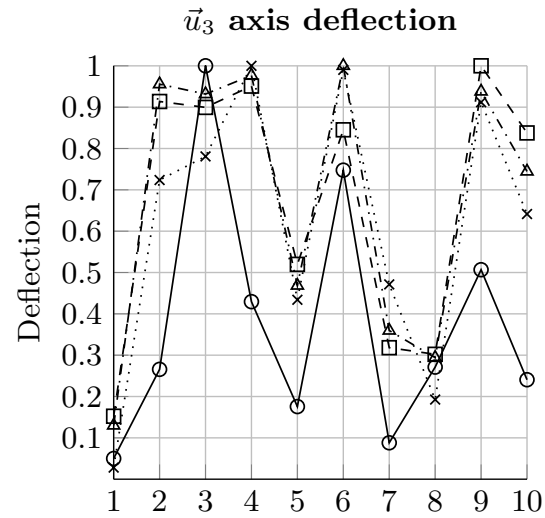

(c) Test Pose $\vec{u}_{2}$ axis deflection

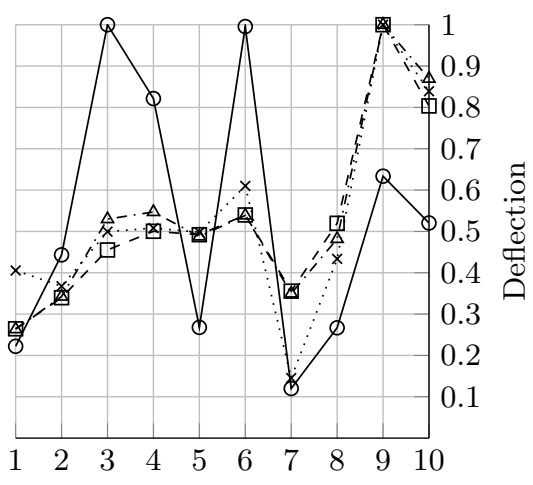

(b) Test Pose

Total Deflection

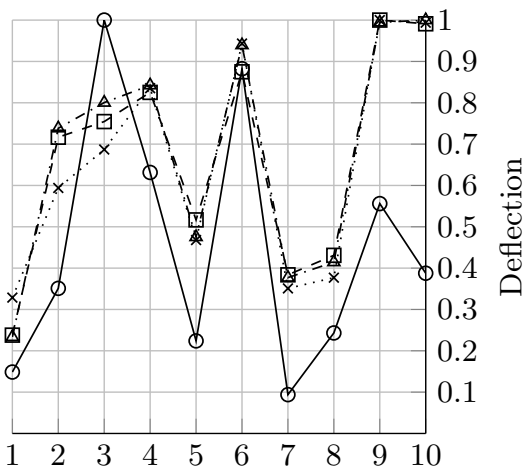

(d) Test Pose

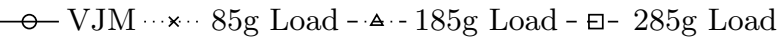

Fig. 6. Normalized VJM and experimental deflections.

Fig. 6 c) shows deflections along $\vec{u}_{3}$. Experimental results of TP2 highly differ in terms of general behavior that needs careful attention. Also, TP2, TP4, TP9, and TP10 have high relative errors between experiment and VJM results.

Finally, overall deflections are illustrated in Fig. 6. In VJM, TP3 has its highest deflection while experiments show that maximum deflection occurs at TP9. Besides, TP3 has a lower value for experiments than TP4 while TP3 in VJM is the most compliant pose. In overall deflections, the dominant axis is $\vec{u}_{3}$. TP6 for all poses, however, is the best fitting pose with the VJM results. 


\section{Conclusion}

In this study, experimental test setup and procedure are described to measure the compliant deflections of a mechanism. The results of these test are used for VJM stiffness model verification that was previously procured.

VJM and experimental results are compared in normalized space to validate the analytical model. Except for a few test poses, VJM and experimental results have a similar stiffness behavior. One of the reasons for the discrepancy of the calculated and measured data is the weight of the laser range sensor, which is not taken into account in the simulation test with the VJM model. Another reason is the assumption that the orientation of the mobile platform does not change during the application of different loads. One last reason is the flexion of the base of the manipulator since it is not produced from high-strength material. Nevertheless, the experimental test setup and its procedure are proposed in this study. The future work includes the addition of an inclinometer to the mobile platform to measure the orientation change and fix the laser range sensor reading accordingly, including sensor weight to the VJM model, and carrying out the experimentation with the actual prototype of the mechanism produced from aluminum material and carbon composite tubes.

Acknowledgement. The study is supported in part by The Scientific and Technological Research Council of Turkey via grant number 117M405. Also, the first author wishes to thank ACRI association for a grant which supported him for a period of study at LARM in Cassino, Italy within 2018. The paper presents results from the research activities of the project ID 37_215, MySMIS code 103415 "Innovative approaches regarding the rehabilitation and assistive robotics for healthy ageing" co-financed by the European Regional Development Fund through the Competitiveness Operational Programme 2014-2020, Priority Axis 1, Action 1.1.4, through the financing contract 20/01.09.2016, between the Technical University of Cluj-Napoca and ANCSI as Intermediary Organism in the name and for the Ministry of European Funds.

\section{References}

1. Kern, T.A.: Engineering haptic devices: a beginner's guide for engineers. Springer Publishing Company, Incorporated (2009)

2. Pinto, C., Corral, J., Altuzarra, O., Hernández, A.: A methodology for static stiffness mapping in lower mobility parallel manipulators with decoupled motions. Robotica 28(5), 719-735 (2010)

3. Carbone, G.: Stiffness analysis and experimental validation of robotic systems. Frontiers of Mechanical Engineering 6(2), 182-196 (2011)

4. Yuan, H., Courteille, E., Deblaise, D.: Elastodynamic analysis of cable-driven parallel manipulators considering dynamic stiffness of sagging cables. In: Robotics and Automation (ICRA), 2014 IEEE International Conference on, pp. 4055-4060. IEEE (2014)

5. Schwenke, H., Knapp, W., Haitjema, H., Weckenmann, A., Schmitt, R., Delbressine, F.: Geometric error measurement and compensation of machinesan update. CIRP Annals 57(2), 660-675 (2008)

6. Nyce, D.S.: Position Sensors. John Wiley \& Sons (2016) 
7. Taner, B., Dede, M.I.C.: Image processing based stiffness mapping of a haptic device. In: New Advances in Mechanisms, Mechanical Transmissions and Robotics, pp. 447-454. Springer (2017)

8. Švaco, M., Šekoranja, B., Šuligoj, F., Jerbić, B.: Calibration of an industrial robot using a stereo vision system. Procedia Engineering 69, 459-463 (2014)

9. Slavković, N.R., Milutinović, D.S., Kokotović, B.M., Glavonjić, M.M., Živanović, S.T., Ehmann, K.F.: Cartesian compliance identification and analysis of an articulated machining robot. FME Transactions 41(2), 83-95 (2013)

10. Hernández-Martínez, E.E., Ceccarelli, M., Carbone, G., López-Cajún, C.S., Jáuregui-Correa, J.C.: Characterization of a cable-based parallel mechanism for measurement purposes\#. Mechanics based design of structures and machines 38(1), 25-49 (2010)

11. Zhang, X., Yang, W., Cheng, X., Chen, Y.: Stiffness identification for serial robot manipulator based on uncertainty approach. In: International Conference on Intelligent Robotics and Applications, pp. 378-388. Springer (2011)

12. Klimchik, A., Pashkevich, A., Wu, Y., Caro, S., Furet, B.: Design of calibration experiments for identification of manipulator elastostatic parameters. arXiv preprint arXiv:1211.6101 (2012)

13. Görgülü, İ., Dede, M.: Computation time efficient stiffness analysis of the modified r-cube mechanism. In: The International Conference of IFToMM ITALY, pp. 231239. Springer (2018)

14. Li, W., Gao, F., Zhang, J.: R-cube, a decoupled parallel manipulator only with revolute joints. Mechanism and Machine Theory 40(4), 467-473 (2005)

15. Gosselin, C.: Stiffness analysis of parallel mechanisms using a lumped model. Int. J. Robotics Automat. 17, 17-27 (2002)

16. Pashkevich, A., Chablat, D., Wenger, P.: Stiffness analysis of overconstrained parallel manipulators. Mechanism and Machine Theory 44(5), 966-982 (2009) 